\title{
Genome-wide analysis of heterosis-related genes in non-heading Chinese cabbage
}

\author{
Hankuil Yi $\cdot$ Jeongyeo Lee $\cdot$ Hayong Song $\cdot$ Xiangshu Dong $\cdot$ Yoonkang Hur
}

Received: 18 September 2017 / Revised: 19 September 2017 / Accepted: 21 September 2017

(c) Korean Society for Plant Biotechnology

\begin{abstract}
Heterosis or hybrid vigor describes a phenomenon that superior phenotypes compared to the two parents are observed in the heterozygous $\mathrm{F}_{1}$-hybrid plants. Identification and characterization of heterosis-related genes (HRGs) will facilitate hybrid breeding in crops. To identify HRGs in Brassica rapa, we analyzed transcriptome profiling using a Br300K microarray in non-heading Chinese cabbage at three developmental stages. A large number of genes were differentially expressed in $\mathrm{F}_{1}$ hybrids and non-additive expression was prominent. Genes that are expressed specifically for $F_{1}$ hybrid at all three stages were Brassica-specific uncharacterized genes and several defense-related genes. Expression of several photosynthesis- and stress-related genes were also $F_{1}$ hybrid-specific. Thirteen NBS-LRR class genes showed high and specific expression in $F_{1}$ hybrid Shulu: some of them were characterized as defense genes in Arabidopsis, but most have not been. Further characterization of these defense-related genes in Brassica species and its application will be helpful for understanding the role of defense responses in heterosis. In addition, results obtained in this study will be valuable to develop molecular markers for heterosis and disease resistance in B. rapa.
\end{abstract}

Keywords Heterosis, $F_{1}$ hybrid, Non-heading Chinese cabbage, NBS-LRR, microarray

${ }^{\dagger}$ Contributed equally

H. Yi ${ }^{\dagger} \cdot$ J. Lee ${ }^{\dagger} \cdot$ H. Song $\cdot$ Y. Hur $(\triangle)$

Department of Biological Sciences, College of Biological Science and Biotechnology, Chungnam National University, Daejeon

34134, Republic of Korea

e-mail: ykhur@cnu.ac.kr

J. Lee

Korea Research Institute of Bioscience and Biotechnology, 125

Gwahangno, Yuseong-gu, Daejoen 34141, Republich of Korea

X. Dong

School of Agriculture, Yunnan University, Kunming, Yunnan, China

\section{Introduction}

Hybrid vigor or heterosis is a phenomenon in which the heterozygous $\mathrm{F}_{1}$-hybrid plants exhibit superior phenotypes in biomass, growth, size and stress resistance, over their homozygous parent inbred lines. Due to superior phenotypes of $F_{1}$ hybrids, the heterosis has been widely used in the commercial seed production of crops, like maize and rice, and vegetable cultivar like Chinese cabbage (Basunanda et al. 2010; Schnable and Springer 2013; Fu et al. 2015; Kawamura et al. 2016; Jeong et al. 2017). Elucidation and application of the heterosis mechanism will help to develop breeding strategies to enhance crop productivity.

The genetic analyses of $F_{1}$ hybrids in maize and rice to understand heterosis mechanism revealed that a large number of QTLs contribute to superior phenotypes of the hybrids in complex manners (Baranwal et al. 2012; Chen 2013; Groszmann et al. 2013; Schnable and Springer 2013). Gene interactions, such as dominance, overdominance, pseudooverdominance, and epistasis, have therefore been suggested to explain heterosis phenotypes (Lippman and Zamir 2007; Charlesworth et al. 2009). Recent molecular analyses of transcriptomes, proteomes, and metabolomes with two parents and hybrids have led to the appreciation of new aspects on the establishment of hybrid vigor, such as epigenetic effects (Groszmann et al. 2011 and 2013; Baranwal et al. 2012; Schnable and Springer 2013; Li et al. 2016; Saeki et al. 2016)].

Transcriptome-wide gene expression study is one of methods to dissect the heterosis at the gene expression level. The initial studies insisted that the increased gene expression level in the hybrids may contribute to heterosis (Romagnoli et al. 1990; Tsaftaris 1995). More recent studies have suggested that heterosis is due to the relative frequencies of genes showing additive and non-additive expression in hybrids (Auger et al. 2005; Guo et al. 2006; Stupar et al. 2008). Additive expression occurs when the hybrid expression 
level is equivalent to the mid-parent values while nonadditive expression occurs whenever the hybrid expression level deviates from the mid-parent level (Stupar et al. 2006). The expression levels outside the parental range in nonadditive expression will greatly contribute heterosis phenotypes. However, it is challenging to determine how many genes or what kinds of genes are involved in heterosis, because large numbers of genes differentially contribute to the superior performance of hybrid, depending on growth stages or organs (Schnable and Springer 2013).

Epigenetic change in protein coding genes and rDNA genes by small RNAs, DNA methylation and chromatin remodeling results in the alteration of gene expression by reprogramming of interacting genomes in hybrids (Chen 2013; Greaves et al. 2015). In Arabidopsis, the role of DNA methylation in hybrid vigor, maintained by METHYLTRANSFERASE 1 (MET1) or DNA METHYLATION 1 (DDM1), has been reported (Shen et al. 2012; Kawanabe et al. 2016). In addition, alteration of circadian rhythms affected growth vigor and biomass increase via change in expression timing of CIRCADIAN CLOCK ASSOCIATED 1 (CCA1) and LATE ELNGATED HYPOCOTYL (LHY) in Arbiosopsis and maize (Ni et al. 2009; Miller et al. 2015; Ko et al. 2016). Particularly, the activation of morningphased genes in hybrids by circadian genes promoted photosynthesis and growth vigor. The early shift of CCA1binding to photosynthesis-and metabolism-associated genes in the morning leads to additive and non-additive expressions, which in turn establish and maintain heterosis (Ko et al. 2016). A transcription factor PHYTOCHROME-INTERACTING FACTOR 4 (PIF4) also plays an important role in hybrid vigor by altering auxin biosynthesis and auxin responsive genes (Wang et al. 2017).

Brassica rapa ssp. chinenssis $F_{1}$ cultivar, Shulu, was bred by cross between Aijiaohung self-incompatible line as female parent and Suzhouqing inbred line as male parent (Hou et al. 2005). The Shulu exhibited improved quality in leaf morphology, disease resistance and yield (Hou et al. 2005), providing a good material to identify growth promoting genes and disease-resistant genes in heterosis. In this study, we applied genome-wide transcript profiling to obtain gene expression information during heterosis in this non-heading Chinese cabbage. Key observations include differential expression of known heterosis-genes in other plants and diseaseresistant genes in hybrids.

\section{Materials and Methods}

Plant materials

Seeds for Brassica rapa ssp. chinenssis $\mathrm{F}_{1}$ cultivar, Shulu
(25081), female parent Aijiaohung (25080) and male parent Suzhouqing (25083) were obtained from Korea Brassica rapa Genome Resource Bank (KBGRB), Chungnam National University, Republic of Korea. Seeds were sown in greenhouse at Chungnam National University, growing from April to May and sampled at indicated time. Shoots from 5 plants for each line were sampled at 15 and 30 DAGs, and frozen in liquid nitrogen until use. For 60 DAG plants, one young and mature leaf from one individual plant were taken from 5 independent plants.

RNA isolation and hybridization to the $\mathrm{Br} 300 \mathrm{~K}$ microarray GeneChip

Total RNA was isolated from samples using TRIzol reagent (Invitrogen, USA), and further purified with a NucleoSpin RNA Clean-up Kit (Macherey-Nagel GmbH \& Co., Germany). For biological repeats, RNA extracted from two independent samples was used in microarray experiments. Microarray experiments and subsequent analyses were performed as described previously (Dong et al. 2013; Song et al. 2017).

Brassica rapa 300K Microarray (Br300K microarray), version 2.0, was composed of 47,548 unigenes as follows: seven 60-nt long probes were designed from each gene, covering $150 \mathrm{bp}$ in the $3^{\prime}$ region of the gene starting from $60 \mathrm{bp}$ upstream of the stop codon with $15 \mathrm{bp}$ shifting. After the hybridization with MAUI chamber (Biomicro, USA), themicroarray was scanned with Genepix 4000 B (Axon, USA) preset with a $5 \mu \mathrm{m}$ resolution for Cy 3 signal. Signals were digitized and analyzed by Nimblescan (Nimblegen, USA). The normal distribution of $\mathrm{Cy} 3$ intensities (prove intensity, PI) was tested with qqline. The data was normalized and processed with cubic spline normalization using quantiles to adjust signal variations between chips, and Rubust Multi-Chip Analysis (RMA) using a median polish algorithm implemented in NimbleScan (Workman et al. 2002; Irizarry et al. 2003).

\section{RT-PCR analysis}

Total RNA $(1 \mu \mathrm{g})$ from each sample was used in cDNA synthesis with the Ace-a kit and the Oligo (dT) primers (Toyobo, Japan). cDNA was diluted 10-fold, and $1 \mu \mathrm{l}$ of diluted cDNA was used in a $20 \mu \mathrm{l}$ PCR reaction. RT-PCR primers are listed in Supplementary Table 1; primer sequences for $B r A C T 2$, used as a control, were 5'- GAACCGGGTG CTCCTCAGGA-3' (forward) and 5' - ATGGTACCGGAATG GTCAAGGC-3' (reverse). A standard PCR was performed with a 5 min denaturation at $94^{\circ} \mathrm{C}$, followed by 25 cycles of $94^{\circ} \mathrm{C}$ for $30 \mathrm{~s}, 55^{\circ} \mathrm{C}$ for $30 \mathrm{~s}$, and $72^{\circ} \mathrm{C}$ for $90 \mathrm{~s}$. PCR 
products were analyzed by electrophoresis through $1.2 \%$ agarose gels.

\section{Results and Discussion}

Description of non-heading Chinese cabbage samples

As mentioned by Hou et al. 2005, growth of $F_{1}$ cultivar Shulu is faster than its parents (Fig. 1). Particularly, selfincompatible female parent Aijiaohung grew slower than male parent Suzhouqing inbred line even at 60 DAG. Significant difference in growth phenotype between two parents, as well as between $F_{1}$ hybrids and either parent, implies that a large number of genes are differentially expressed.
Analysis of Br300K microarray

To identify heterosis-related genes (HRGs), we performed Br300K microarray experiment twice with 9 samples collected at 3 stages (15 DAG, 30 DAG and 60 DAG) and results were summarized in Supplementary Table 1. As expected, a large number of genes were differently expressed (Table 1). Regarding to DEGs, the largest number was detected at 15 DAG samples or between two parents. These results indicated that the early stage of growth may determine the heterosis phenotypes in $F_{1}$ cultivar Shulu. The highest difference between two parents also expected that the genetic difference on the gene expression regulation will be different from those obtained from heading-type Chinese cabbage, in which they have used similar parents (Saeki et al. 2016).
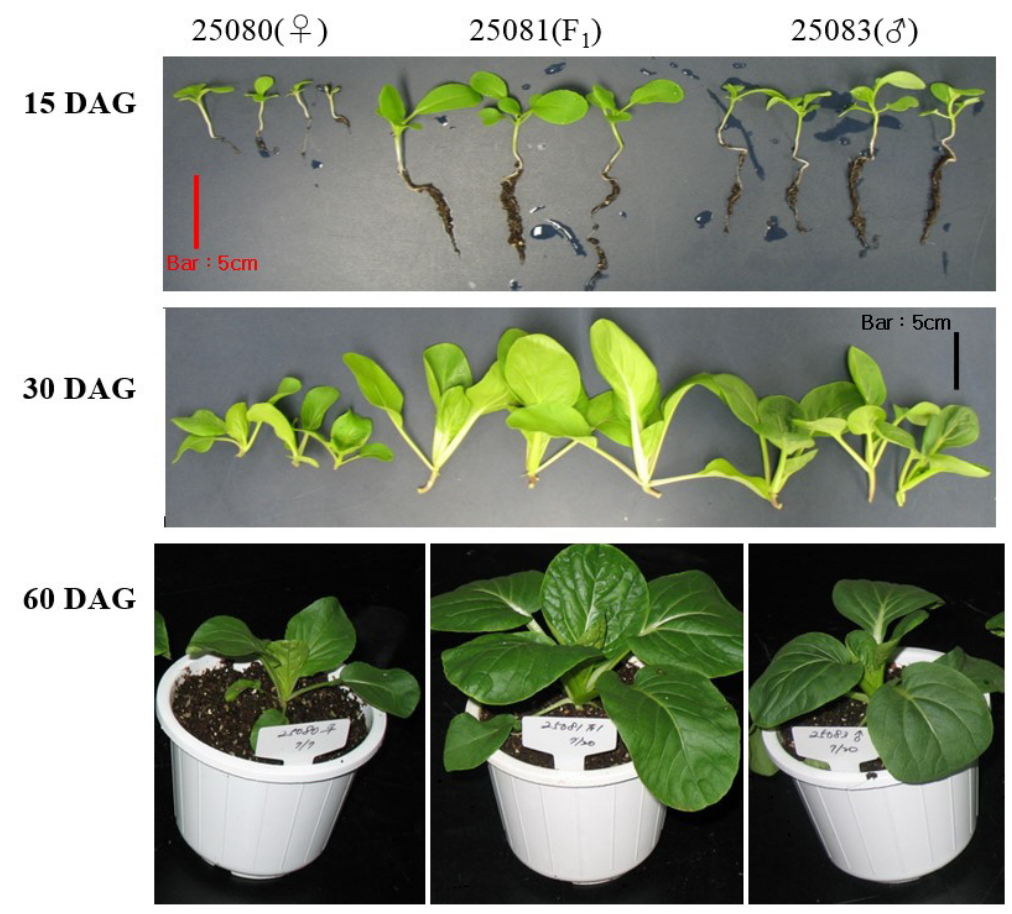

Fig. 1 Morphology of plants used in this experiment. Self-incompatible Aijiaohung line (25080) and Suzhouqing inbred line (25083) were used as female parent male parent, respectively. '25081' indicates $F_{1}$ hybrid

Table 1 Summary of Br300K microarray results. DEGs represent differentially expressed genes over two-fold between two Chinese cabbage lines. For non-additive expression, genes showing over 1.5 -fold difference between $\mathrm{F}_{1}$ hybrid and mid-parent values were counted. 80,81 and 83 represented abbreviation of 25080, 25081 and 25083, respectively

\begin{tabular}{|c|c|c|c|c|c|c|c|c|c|c|}
\hline \multirow{2}{*}{\multicolumn{2}{|c|}{ Classification }} & \multicolumn{3}{|c|}{$15 \mathrm{DAG}$} & \multicolumn{3}{|c|}{$30 \mathrm{DAG}$} & \multicolumn{3}{|c|}{$60 \mathrm{DAG}$} \\
\hline & & $81 / 80$ & $81 / 83$ & $80 / 83$ & $81 / 80$ & $81 / 83$ & $80 / 83$ & $81 / 80$ & $81 / 83$ & $80 / 83$ \\
\hline \multirow[t]{2}{*}{ DEGs } & Up & 1,714 & 2,060 & 2,882 & 2,462 & 1,165 & 2,415 & 1,412 & 1,730 & 2,749 \\
\hline & Down & 1,557 & 3,523 & 4,295 & 1,736 & 1,239 & 3,423 & 1,528 & 1,638 & 2,404 \\
\hline \multicolumn{2}{|c|}{ Additive expression } & \multicolumn{3}{|c|}{$815(41)$} & \multicolumn{3}{|c|}{$952(33)$} & \multicolumn{3}{|c|}{$808(50)$} \\
\hline \multicolumn{2}{|c|}{ Non-additive expression } & \multicolumn{3}{|c|}{$13,695(6,900)$} & \multicolumn{3}{|c|}{$14,358(7,918)$} & \multicolumn{3}{|c|}{$12,692(5,561)$} \\
\hline
\end{tabular}

( ) : number of genes showing over 1,000 in PI values, which has been considered as significant levels of transcripts. 
Expression pattern analysis revealed that non-additive expression was prominent compared with additive expression in non-heading type (Table 1), different from the previous results for heading type Chinese cabbage (B. rapa ssp. pekinensis) (Saeki et al. 2016). Saeki et al. (20106) reported that most genes show an additive expression pattern, and any expression level differences between parental lines were maintained in $F_{1}$ hybrids. This might be caused by somewhat similar phenotypes between parents used for $\mathrm{F}_{1}$ hybrid production in the heading type Chinese cabbage. Two parents used in this study showed quite different phenotypes, such as growth (Fig. 1) and others (Hou et al. 2005), leading to non-additive expression in most genes. Conflicting results were also reported in previous maize F1 studies. In one study, the majority $(\sim 75 \%)$ of genes exhibited additive expression in the hybrid and only small numbers of the non-additively expressed genes exhibited expression levels outside the parental range (Guo et al. 2006; Stupar and Springer 2006). In another study, much higher levels of non-additive expression and numerous examples of expression outside the parental range were observed (Auger et al. 2005; Meyer et al. 2007). It is not clear whether these differences are caused by biological differences between tissues, genotypes, or differences in the expression profiling platforms.

\section{Identification of putative HRGs}

It is not easy to determine which genes and how many genes are involved in heterosis, because large numbers of genes with complex roles are involved (Schnable and Springer 2013). We hypothesized that HRGs will be differentially expressed in $F_{1}$ hybrids compared to both parents: up-regulated or down-regulated. Consistent with our hypothesis up- and down-regulation of many genes were detected in $F_{1}$ hybirds (Fig. 2). However, only 14 genes and 1 gene were found to be up-regulated and down-regulated at all growth stages, respectively. These small numbers of putative HRGs might be explained, if developmental stage-dependent complex and different actions by large numbers of genes or alleles are required for heterosis (Schnable and Springer 2013). We further speculated that genes differently expressed at two different stages are HRGs with more important roles in heterosis. It turned out that there are 23 up-regulated DEGs overlapping between 15 and 30 DAG, another 23 DEGs between 30 and 60 DAG, and 124 DEGs between 15 and 60 DAG. In similar approach for down-regulated DEGs, 9 between 15 and 30 DAG, 18 between 30 and 60 DAG, and 51 between 15 and 60 DAG were identified. These DEGs, especially expressed in the early stages, were
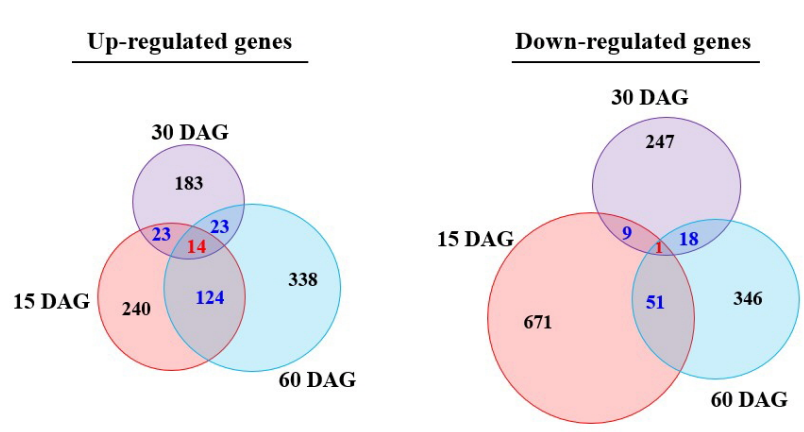

Fig. 2 Distribution of DEGs in $F_{1}$ hybrids showing more than two fold expression change, when compared with both parents. DAG, days after germination

considered as HRG candidates for non-heading Chinese cabbage.

Among DEGs shown in Figure 2, we further analyzed (1) genes that were up-regulated in all stages, (2) upregulated in both 15 and 30 DAGs, (3) up-regulated in both 30 and 60 DAGs and (4) down-regulated in all stages, as HRG candidates (Table 2). Interestingly, most up-regulated genes belonged to predicted mRNAs or uncharacterized Brassica mRNAs, suggesting that heterosis of B. rapa $\mathrm{ssp}$ chinenesis is affected by Brassica-specific genes. Still, we found several genes, which might possess heterosis-related functions, in the list: Brapa_ESTC051755 for photosynthesis and Brapa_ESTC019040 for nucleic acid synthesis were up-regulated in all stages. In addition, 4 putative defenserelated genes were found in up-regulated genes: Brapa ESTC031864 encoding leucine-rich repeat protein kinase family protein, Brapa ESTC002203 encoding 20S PROTEASOME BETA SUBUNIT PBF1, Brapa_ESTC014631 encoding NAD(P)-linked oxidoreductase superfamily protein and Brapa ESTC024642 encoding disease resistance-responsive dirigent-like protein. Kumar et al. (2016) reported that Arabidopsis orthologs for Brapa_ESTC014631 and Brapa_ESTC024642 exhibit defense response. For down-regulated genes in all stages, only one gene, Brapa_ESTC012945, which encodes bifunctional inhibitor/lipid-transfer protein/seed storage $2 \mathrm{~S}$ albumin superfamily protein, was identified. The function of this protein has not been identified and requires functional studies.

Expression of genes showing homology to HRGs identified in other plants

Circadian rhythm-, chromatin remodeling- and stress responsive-related genes have been reported as HRGs in Arabidopdopsis thaliana, and photosynthesis-related gene showed up-regulation in B. rapa $\mathrm{F}_{1}$ hybrid (references in Table 3). We summarized expression levels of $B$. rapa homolgos of 
Table 2 List of differentially expressed genes in $F_{1}$ hybrid compared to its parent line over two fold. Genes showing over two fold up-regulation at least two stages of Chinese cabbage growth (15 DAG, 30 DAG, and 60 DAG) and down-regulated in all stages were selected for analysis (see Fig. 2)

\begin{tabular}{|c|c|c|c|c|c|c|c|c|c|c|}
\hline \multirow{3}{*}{$\begin{array}{l}\text { Up/Down } \\
\text { (Stages) }\end{array}$} & \multirow{3}{*}{ At_Locus } & \multirow{3}{*}{ Br_SEQ ID } & \multirow{3}{*}{ BRADID } & \multirow{3}{*}{ Description, BlastN } & \multicolumn{6}{|c|}{ Fold change } \\
\hline & & & & & \multicolumn{2}{|c|}{$15 \mathrm{DAG}$} & \multicolumn{2}{|c|}{$30 \mathrm{DAG}$} & \multicolumn{2}{|c|}{$60 \mathrm{DAG}$} \\
\hline & & & & & $81 / 80$ & $81 / 83$ & $81 / 80$ & $81 / 83$ & $81 / 80$ & $81 / 83$ \\
\hline \multirow[t]{14}{*}{$\begin{array}{l}\text { UP } \\
\text { (All) }\end{array}$} & No_hits_found & Brapa_ESTCO48713 & Bra006663 & PREDICIED. B. rapa QWRF motif-containing protein 7-like & 18.4 & 138.0 & 15.1 & 205.1 & 25.5 & 227.8 \\
\hline & & Brapa_ESTC048714 & - & 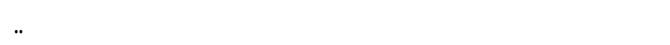 & 5.6 & 8.8 & 6.7 & 9.4 & 9.5 & 9.1 \\
\hline & AT1G49980 & Brapa_ESTC019040 & Bra018822 & DNARNA polymerases superfamily protein & 57.5 & 51.2 & 40.3 & 40.3 & 47.8 & 41.6 \\
\hline & No hits_found & Brapa_ESTC048297 & - & PREDICIED: Brassica rapa uncharacterized LOC103834461 & 37.1 & 34.6 & 15.3 & 17.0 & 103.1 & 141.1 \\
\hline & & Brapa_ESTCO48298 & - & .. & 3.3 & 2.8 & 3.5 & 4.9 & 28.8 & 24.2 \\
\hline & No hits_found & Brapa_ESTC049333 & - & $\mathrm{Br}$ sequence & 30.4 & 25.7 & 29.3 & 28.7 & 35.5 & 29.9 \\
\hline & AT5G47110 & Brapa_ESTC051755 & Bra023915 & At5g47110 gene (light-harvesting-like protein 3 (LIL3)) & 28.5 & 32.6 & 15.5 & 15.2 & 58.1 & 51.3 \\
\hline & No hits_found & Brapa_ESTCO42529 & Bra038041 & PREDICTED: B. napus uncharacterized LOC106433243 & 24.4 & 4.5 & 8.1 & 2.8 & 11.8 & 11.2 \\
\hline & No hits_found & Brapa_ESTC051614 & Bra010245 & PREDICIED: B. napus uncharacterized LOC106418084, ncRNA & 21.9 & 26.2 & 9.7 & 9.4 & 46.2 & 2.7 \\
\hline & No hits_found & Brapa_ESTCO01522 & - & $\mathrm{Br}$ sequence & 19.4 & 21.6 & 23.1 & 9.8 & 42.2 & 2.1 \\
\hline & AT2G13970 & Brapa_ESTCO44073 & Bra038041 & PREDICTED: B. napus uncharacterized LOC106431633 & 11.6 & 3.8 & 6.4 & 3.8 & 11.4 & 10.0 \\
\hline & АТЗС60820 & Brapa_ESTCOO2203 & Bra014468 & 20S PROTEASOME BETA SUBUNT PBF1 & 6.9 & 7.3 & 7.8 & 7.9 & 7.2 & 33.0 \\
\hline & No hits_found & Brapa_ESTCO49334 & - & $\mathrm{Br}$ sequence & 3.5 & 6.1 & 4.5 & 4.8 & 7.4 & 7.3 \\
\hline & No hits found & Brapa ESTC031120 & Bra027213 & PREDICIED. B. napus putative F-box protein At3g17620 & 2.8 & 3.7 & 3.4 & 2.9 & 5.0 & 4.2 \\
\hline \multirow[t]{9}{*}{$\underset{(15 / 30)}{U p}$} & No hits_found & rapa_ESTC051613 & Bra010245 & PREDICTED: B.a rapa uncharacterized LOCl03849825 & 42.9 & 48.9 & 14.6 & 18.0 & 34.1 & 1.5 \\
\hline & No hits_found & Brapa_ESTC033737 & Bra006426 & PREDICIED: B. rapa uncharacterized LOC103856270 & 17.6 & 29.0 & 32.1 & 25.8 & 29.7 & 0.9 \\
\hline & No hits_found & Brapa_ESTCO03869 & Bra013078 & PREDICIED: B. rapa uncharacterized LOC103856270 & 15.0 & 7.3 & 18.0 & 10.1 & 25.4 & 0.5 \\
\hline & No hits_found & Brapa_ESTCO03723 & Bra004000 & PREDICIED. B. rapa probable protein S-acyltransferase 22 & 12.7 & 2.6 & 6.3 & 3.6 & 1.6 & 2.0 \\
\hline & No hits_found & Brapa_ESTCO04680 & $\mathrm{Bra} 019236$ & PREDICIED. B. rapa probable protein S-acyltransferase 22 & 8.3 & 7.7 & 6.3 & 7.8 & 1.8 & 2.1 \\
\hline & AT5G19760 & Brapa_ESTC011203 & Bra006516 & Mitochondrial substrate carrier family protein & 2.0 & 2.2 & 2.1 & 2.2 & 1.9 & 2.0 \\
\hline & AT1G60710* & Brapa_ESTC014631 & Bra027140 & NAD(P)-linked oxidoreductase superfamily protein (ATB2)(Auxin-inducible) & 21.1 & 35.7 & 8.0 & 9.7 & 14.7 & 1.0 \\
\hline & No hits_found & Brapa_ESTC033736 & Bra013078 & PREDICIED: B. rapa uncharacterized LOC103856270 & 11.0 & 3.0 & 22.6 & 6.9 & 16.4 & 0.5 \\
\hline & No hits_found & Brapa_ESTCO43214 & Bra031342 & $\begin{array}{l}\text { PREDICTED: B. napus splicing factor U2af large subunit B-like } \\
\text { (LOC106346061) }\end{array}$ & 6.9 & 2.2 & 9.8 & 3.5 & 14.6 & 0.7 \\
\hline \multirow[t]{9}{*}{$\begin{array}{c}\text { Up } \\
(30 / 60)\end{array}$} & AT3G52860 & Brapa_ESTCO01902 & Bra016859 & Arabidopsis thaliana other RNA lncRNA & 1.0 & 1.4 & 2.1 & 8.6 & 2.7 & 4.2 \\
\hline & AT4G13760 & Brapa_ESTCO20421 & Bra000327 & Pectin lyase-like superfamily protein & 1.3 & 2.5 & 5.3 & 5.0 & 11.4 & 9.7 \\
\hline & AT1G64160* & Brapa_ESTCO24642 & Bra027681 & Disease resistance-responsive (dirigent-like protein) family protein (DIR5) & 2.2 & 0.5 & 8.0 & 2.5 & 3.1 & 2.4 \\
\hline & AT3G47090 & Brapa_ESTC031864 & Bra035694 & Leucine-rich repeat protein kinase family protein (LRR-RLK) & 1.4 & 0.7 & 12.3 & 18.1 & 11.6 & 11.0 \\
\hline & No hits_found & Brapa_ESTC038099 & - & PREDICIED: B. napus uncharacterized LOC106386659, ncRNA & 0.3 & 2.6 & 3.4 & 6.3 & 2.3 & 2.9 \\
\hline & No hits_found & Brapa_ESTCO40600 & Bra035021 & PREDICTED: B. napus uncharacterized LOC106403229 & 2.1 & 1.4 & 2.1 & 2.6 & 4.5 & 4.2 \\
\hline & AT1G43090 & Brapa_ESTCO41995 & Bra017014 & Pectin lyase-like superfamily protein & 0.7 & 0.6 & 22.1 & 10.4 & 31.3 & 38.2 \\
\hline & No hits_found & Brapa_ESTC051749 & - & PREDICTED: B. napus uncharacterized LOCI06433400 & 1.8 & 1.3 & 7.2 & 3.7 & 7.2 & 4.1 \\
\hline & No hits found & Brapa ESTC051750 & - & PREDICTED: B. napus uncharacterized LOC106433400 & 1.7 & 1.2 & 14.3 & 8.9 & 16.0 & 13.6 \\
\hline $\begin{array}{l}\text { Down } \\
\text { (All) }\end{array}$ & AT1G62510 & Brapa_ESTC012945 & Bra027039 & $\begin{array}{l}\text { Bifunctional inhibitor/lipid-transfer protein/seed storage } 2 \mathrm{~S} \text { albumin } \\
\text { superfamily protein }\end{array}$ & -5.0 & -4.3 & -2.4 & -7.7 & -3.8 & -7.8 \\
\hline
\end{tabular}

List of differentially expressed genes in $F_{1}$ hybrid compared to its parent line over two fold. Genes showing over two fold up-regulation at least two stages of Chinese cabbage growth (15 DAG, 30 DAG, and 60 DAG) and down-regulated in all stages were selected for analysis (see Fig. 2)(No_hit_found: no Arabidopsis homolog present, - : no cDNA found in BRAD, *: gene identified by Kumar et al. (2016) as defense role against cotton bollworm.)

these known HRGs in non-heading Chinese cabbage (Table 3 ). According to probe intensity (PI) values, $L H C A 2$ and stress-responsive genes, such as COR78 and COR47, appeared to be HRGs in non-heading cabbage, while conclusion for others were not clear. This results implies that heterosis mechanisms are complex and species-specific. In Arabidopsis, critical roles of PIF4, CCA1 and LHY in heterosis were reported (Shen et al. 2012; Miller et al. 2015; Ko et al. 2016), but their roles in non-heading Chinese cabbage are not supported by mRNA expression analysis. In maize, the early activation of morning-phased genes by $C C A 1$ in the maize hybrids promotes photosynthesis and growth vigor (Ko et al. 2016). This temporal shift of ZmCCA1-binding targets correlated with non-additive and additive gene ex- 
Table 3 Expression of B. rapa genes homologous to genes showing heterosis-related expression in other plants

\begin{tabular}{|c|c|c|c|c|c|c|c|c|c|c|c|c|}
\hline \multirow{3}{*}{ At_Locus } & \multirow{3}{*}{ Gene description } & \multirow{3}{*}{ Br_SEQ Id } & \multicolumn{9}{|c|}{ Probe intensity } & \multirow{3}{*}{ Reference } \\
\hline & & & \multicolumn{3}{|c|}{$15 \mathrm{DAG}$} & \multicolumn{3}{|c|}{$30 \mathrm{DAG}$} & \multicolumn{3}{|c|}{$60 \mathrm{DAG}$} & \\
\hline & & & 25080 & 25081 & 25083 & 25080 & 25081 & 25083 & 25080 & 25081 & 25083 & \\
\hline \multirow[t]{5}{*}{ AT1G01060 } & \multirow[t]{5}{*}{ LHY (LATE ELONGATED HYPOCOTYL) } & Brapa_ESTC008780 & 480 & 270 & 396 & 307 & 266 & 295 & 1583 & 3235 & 1958 & $\begin{array}{l}\text { Ni et al. } \\
2009\end{array}$ \\
\hline & & Brapa_ESTC027456 & 95 & 39 & 43 & 35 & 77 & 76 & 1457 & 1354 & 1457 & \\
\hline & & Brapa_ESTC028354 & 1531 & 1484 & 960 & 1227 & 870 & 1185 & 3884 & 4653 & 4341 & \\
\hline & & Brapa_ESTC028906 & 509 & 378 & 247 & 321 & 361 & 443 & 2945 & 3187 & 3291 & \\
\hline & & Brapa_ESTC029140 & 220 & 90 & 183 & 100 & 84 & 103 & 1295 & 2145 & 1297 & \\
\hline \multirow[t]{3}{*}{ AT1G01510 } & \multirow[t]{3}{*}{ AN (ANGUSTIFOLIA) } & Brapa_ESTC014581 & 8823 & 10283 & 9862 & 9672 & 12697 & 12208 & 11634 & 13445 & 16742 & $\begin{array}{l}\text { Kim et al. } \\
2002\end{array}$ \\
\hline & & Brapa_ESTC015231 & 4304 & 4017 & 3004 & 4437 & 3812 & 4102 & 5597 & 6491 & 6791 & \\
\hline & & Brapa_ESTC047153 & 3610 & 3984 & 4698 & 3525 & 4169 & 5220 & 3738 & 4304 & 7589 & \\
\hline \multirow[t]{2}{*}{ AT1G22770 } & \multirow[t]{2}{*}{ GI (GIGANTEA) } & Brapa_ESTC017304 & 10582 & 13343 & 17247 & 11276 & 17041 & 16639 & 11515 & 13092 & 15503 & $\begin{array}{l}\mathrm{Ni} \text { et al. } \\
2009\end{array}$ \\
\hline & & Brapa_ESTC039985 & 14641 & 14131 & 17624 & 13652 & 18088 & 17106 & 10937 & 12892 & 13800 & \\
\hline \multirow[t]{2}{*}{ AT2G43010 } & \multirow[t]{2}{*}{ PIF4 (PHYTOCHROME INTERACTING FACTOR 4) } & Brapa_ESTC012017 & 1438 & 2463 & 4227 & 2040 & 3237 & 3449 & 1863 & 2494 & 6262 & $\begin{array}{l}\text { Wang et al. } \\
2017\end{array}$ \\
\hline & & Brapa_ESTC018786 & 7303 & 8497 & 11878 & 8071 & 10934 & 12229 & 6198 & 8463 & 16081 & \\
\hline \multirow[t]{2}{*}{ AT2G46830 } & \multirow[t]{2}{*}{ CCA1 (CIRCADIAN CLOCK ASSOCIATED 1) } & Brapa_ESTC006966 & 617 & 197 & 288 & 84 & 99 & 126 & 1913 & 1194 & 891 & $\begin{array}{l}\text { Ni et al. } \\
2009\end{array}$ \\
\hline & & Brapa_ESTC008890 & 945 & 280 & 309 & 164 & 249 & 166 & 3656 & 2975 & 1858 & \\
\hline \multirow[t]{8}{*}{ AT3G51800 } & \multirow[t]{8}{*}{ ATG2 (G2p-related protein)(EBP1) } & Brapa_ESTC007218 & 8864 & 8878 & 10174 & 10754 & 10830 & 12012 & 12180 & 11249 & 9869 & $\begin{array}{l}\text { Wang et al. } \\
2016\end{array}$ \\
\hline & & Brapa_ESTC007283 & 16033 & 14047 & 15178 & 18170 & 18601 & 20514 & 21149 & 19476 & 19020 & \\
\hline & & Brapa_ESTC008892 & 14493 & 14210 & 14921 & 17826 & 15503 & 18299 & 17708 & 18251 & 16788 & \\
\hline & & Brapa_ESTC017696 & 6663 & 5780 & 6951 & 9232 & 10731 & 10114 & 12313 & 11761 & 12483 & \\
\hline & & Brapa_ESTC020359 & 9573 & 7849 & 11016 & 11578 & 10661 & 11844 & 14132 & 13022 & 12074 & \\
\hline & & Brapa_ESTC021489 & 10939 & 9850 & 13452 & 12292 & 11871 & 13230 & 16308 & 14754 & 13506 & \\
\hline & & Brapa_ESTC025539 & 4032 & 3531 & 6893 & 3853 & 5519 & 5702 & 5197 & 3238 & 2533 & \\
\hline & & Brapa_ESTC043540 & 10325 & 12035 & 11256 & 12677 & 13211 & 14065 & 12451 & 15279 & 13073 & \\
\hline AT4G19020 & CMT2 (Chromomethylase 2) & Brapa_ESTC009064 & 2915 & 3337 & 3167 & 2479 & 2006 & 2499 & 3125 & 3424 & 3223 & $\begin{array}{l}\text { Zemach et al. } \\
\quad 2013\end{array}$ \\
\hline \multirow[t]{2}{*}{ AT5G05450 } & \multirow[t]{2}{*}{ RH18 (DEAD/DEAH box helicase, putative) } & Brapa_ESTC030024 & 2565 & 2964 & 3105 & 2847 & 2102 & 2437 & 2622 & 2540 & 2169 & $\begin{array}{l}\text { Plötner et al. } \\
2017\end{array}$ \\
\hline & & Brapa_ESTC045372 & 6793 & 6539 & 7459 & 7947 & 5535 & 7071 & 7744 & 7255 & 7546 & \\
\hline AT5G49160 & METI (DECREASED METHYLATION 2DNA) & Brapa_ESTC014151 & 6107 & 5762 & 4380 & 8626 & 3442 & 5289 & 6703 & 5063 & 3701 & $\begin{array}{l}\text { Kawanabe et } \\
\text { al. } 2016\end{array}$ \\
\hline AT5G61380 & TOCl (TIMING OF CAB1 1) & Brapa_ESTC002126 & 2773 & 3582 & 4766 & 3066 & 2649 & 3641 & 944 & 766 & 1112 & $\begin{array}{l}\text { Ni et al. } \\
2009\end{array}$ \\
\hline & & Brapa_ESTC006958 & 7045 & 8226 & 9979 & 12056 & 10903 & 10955 & 2257 & 2138 & 3015 & \\
\hline & & Brapa_ESTC033209 & 16000 & 19937 & 20292 & 18860 & 18627 & 19981 & 7451 & 7250 & 9041 & \\
\hline AT5G66750 & DDMI (DECREASED DNA METHYLATION 1) & Brapa_ESTC020388 & 1798 & 1305 & 1356 & 2802 & 953 & 1624 & 2544 & 2018 & 1738 & $\begin{array}{l}\text { Zemach et al. } \\
\quad 2013\end{array}$ \\
\hline & $\begin{array}{l}\text { SWI2/SNF2-like protein; Chromatin remodeling } 1 \\
\text { (CHR1) }\end{array}$ & Brapa_ESTC030555 & 4050 & 2966 & 2595 & 5163 & 2537 & 2736 & 4415 & 3853 & 3338 & \\
\hline AT4G25080 & $\begin{array}{l}\text { CHLM (MAGNESIUM-PROTOPORPHYRIN IX } \\
\text { METHYLTRANSFERASE) }\end{array}$ & Brapa_ESTC002086 & 13691 & 18396 & 14496 & 14269 & 12665 & 13189 & 14831 & 16039 & 14486 & $\begin{array}{l}\text { Saeki et al. } \\
2016\end{array}$ \\
\hline & & Brapa_ESTC015556 & 14397 & 13943 & 11646 & 12510 & 9260 & 8697 & 15347 & 12276 & 9566 & \\
\hline & & Brapa_ESTC047649 & 6196 & 5880 & 6028 & 6742 & 5821 & 3960 & 7479 & 6888 & 7527 & \\
\hline AT3G61470 & $\begin{array}{l}\text { LHCA2 (Photosystem I light harvesting complex } \\
\text { gene 2) }\end{array}$ & Brapa_ESTC007961 & 40044 & 45566 & 36797 & 42413 & 37810 & 41103 & 40901 & 42837 & 37873 & $\begin{array}{l}\text { Saeki et al. } \\
\quad 2016\end{array}$ \\
\hline & & Brapa_ESTC010415 & 38879 & 41868 & 37548 & 36718 & 33981 & 38168 & 37282 & 35083 & 36177 & \\
\hline & & Brapa_ESTC012915 & 22986 & 28174 & 24231 & 22768 & 19967 & 19510 & 24822 & 22579 & 22955 & \\
\hline & & Brapa_ESTC023178 & 3647 & 3505 & 2885 & 2886 & 2833 & 2375 & 2543 & 2964 & 2952 & \\
\hline & & Brapa_ESTC027380 & 4004 & 5096 & 5055 & 2954 & 2592 & 2174 & 5670 & 4264 & 4704 & \\
\hline
\end{tabular}


Table 3 Continued

\begin{tabular}{|c|c|c|c|c|c|c|c|c|c|c|c|c|}
\hline \multirow{3}{*}{ At_Locus } & \multirow{3}{*}{ Gene description } & \multirow{3}{*}{ Br_SEQ Id } & \multicolumn{9}{|c|}{ Probe intensity } & \multirow{3}{*}{ Reference } \\
\hline & & & \multicolumn{3}{|c|}{$15 \mathrm{DAG}$} & \multicolumn{3}{|c|}{$30 \mathrm{DAG}$} & \multicolumn{3}{|c|}{$60 \mathrm{DAG}$} & \\
\hline & & & 25080 & 25081 & 25083 & 25080 & 25081 & 25083 & 25080 & 25081 & 25083 & \\
\hline & & Brapa_ESTC029407 & 23250 & 23845 & 24536 & 19038 & 24518 & 18430 & 23398 & 31942 & 28986 & \\
\hline & & Brapa_ESTC031617 & 102 & 72 & 12 & 195 & 133 & 23 & 168 & 72 & 15 & \\
\hline & & Brapa_ESTC051898 & 90 & 148 & 369 & 71 & 69 & 53 & 409 & 379 & 437 & \\
\hline \multirow[t]{2}{*}{ AT3G22231 } & $\begin{array}{l}\text { PCC1 (PATHOGEN AND CIRCADIAN } \\
\text { CONTROLLED 1) }\end{array}$ & Brapa_ESTC028145 & 121 & 10803 & 12587 & 129 & 5754 & 13964 & 84 & 12832 & 15138 & $\begin{array}{l}\text { Miller et al. } \\
2015\end{array}$ \\
\hline & & Brapa_ESTC047280 & 149 & 12667 & 15914 & 156 & 12142 & 16511 & 34 & 17737 & 24380 & \\
\hline \multirow[t]{8}{*}{ AT2G40000 } & $\begin{array}{l}\text { HSPRO2 (HOMOLOG OF SUGAR BEET HS1 } \\
\text { PRO-2) }\end{array}$ & Brapa_ESTC011718 & 20546 & 11481 & 10834 & 8698 & 8817 & 6869 & 2015 & 2417 & 1754 & $\begin{array}{l}\text { Miller et al. } \\
2015\end{array}$ \\
\hline & & Brapa_ESTC012195 & 19188 & 19022 & 22703 & 10708 & 11129 & 13634 & 5408 & 7166 & 9784 & \\
\hline & & Brapa_ESTC014954 & 6867 & 3389 & 4766 & 2478 & 4776 & 3257 & 1020 & 802 & 1033 & \\
\hline & & Brapa_ESTC021307 & 22562 & 18144 & 22090 & 9919 & 10494 & 11943 & 3862 & 4634 & 8553 & \\
\hline & & Brapa_ESTC021617 & 25259 & 18414 & 13942 & 14969 & 16658 & 11849 & 7868 & 7790 & 6467 & \\
\hline & & Brapa_ESTC024564 & 8822 & 4388 & 5638 & 2509 & 5218 & 4074 & 818 & 627 & 1049 & \\
\hline & & Brapa_ESTC025072 & 16419 & 15051 & 17053 & 8610 & 9287 & 11446 & 4059 & 5230 & 7798 & \\
\hline & & Brapa_ESTC038750 & 23748 & 20199 & 22408 & 12753 & 12716 & 15184 & 5145 & 6741 & 11178 & \\
\hline \multirow[t]{3}{*}{ AT2G14610 } & PR1 (PATHOGENESIS-RELATED GENE 1) & Brapa_ESTC002102 & 24976 & 7546 & 16429 & 19659 & 14660 & 8789 & 14617 & 17595 & 18051 & $\begin{array}{l}\text { Miller et al. } \\
2015\end{array}$ \\
\hline & & Brapa_ESTC013481 & 7451 & 6349 & 6681 & 7800 & 7648 & 8177 & 2569 & 1400 & 1203 & \\
\hline & & Brapa_ESTC042390 & 31300 & 11521 & 21992 & 25887 & 18717 & 12265 & 18855 & 21498 & 21687 & \\
\hline \multirow[t]{2}{*}{ AT5G52310 } & COR78 (COLD REGULATED 78) & Brapa_ESTC003116 & 29897 & 33849 & 29168 & 35598 & 24688 & 30845 & 22402 & 19684 & 19596 & $\begin{array}{l}\text { Miller et al. } \\
2015\end{array}$ \\
\hline & & Brapa_ESTC007756 & 22484 & 26325 & 23312 & 20265 & 22952 & 19805 & 18859 & 22770 & 22197 & \\
\hline \multirow[t]{2}{*}{ AT1G20440 } & COR47 (COLD REGULATED 47) & Brapa_ESTC047060 & 29034 & 29501 & 32189 & 25268 & 21550 & 21905 & 26515 & 26981 & 26378 & $\begin{array}{l}\text { Miller et al. } \\
2015\end{array}$ \\
\hline & & Brapa_ESTC018725 & 19163 & 18060 & 20708 & 15488 & 14960 & 11953 & 17599 & 18980 & 17988 & \\
\hline \multirow[t]{5}{*}{ AT5G25610 } & RD22 (RESPONSIVE TO DESSICATION 22) & Brapa_ESTC001082 & 21734 & 21850 & 20960 & 27979 & 37840 & 37698 & 24547 & 27274 & 28453 & $\begin{array}{l}\text { Miller et al. } \\
2015\end{array}$ \\
\hline & & Brapa_ESTC010889 & 15824 & 12213 & 12688 & 21638 & 17455 & 21897 & 19309 & 16196 & 13363 & \\
\hline & & Brapa_ESTC025981 & 22113 & 20015 & 17356 & 30697 & 39296 & 36578 & 26766 & 25407 & 23135 & \\
\hline & & Brapa_ESTC032166 & 255 & 135 & 52 & 232 & 170 & 121 & 295 & 307 & 121 & \\
\hline & & Brapa_ESTC049783 & 6212 & 5183 & 5883 & 7006 & 8241 & 7307 & 7224 & 6590 & 6255 & \\
\hline
\end{tabular}

pression in early and late stages of seedling development. Because we did not examine expression levels throughout a day, we do not exclude a possibility that expression level of circadian rhythm-related genes correlate to heterosis phenotype in non-heading Chinese cabbage.

\section{Expression of NBS-LRR class genes}

According to original breeders, $F_{1}$ hybrid Shulu shows resistance to various diseases, such as TuMV, downy mildew and alternaria leaf spot (Hou et al. 2005). Therefore, we analyzed expression of disease resistance genes like NBSLRR class. Among 99 NBS-LRR class protein genes included in the $\mathrm{Br} 300 \mathrm{~K}$ microarray, 17 genes corresponding 13 Arabidopsis genes were highly expressed in $\mathrm{F}_{1}$ hybrids (Table 4). Only three Arabidopsis genes have been func- tionally characterized so far. AT5G11250, homolog of Brapa ESTC009435 and Brapa_ESTC032144, is known as BURNOUT1 $(B N T 1)$ and encodes a TIR-NBS-LRR protein responsible for disease resistance (Sarazin et al. 2015). AT5G11250 affects the levels of stress hormones, such as jasmonic acid, salicylic acid, abscisic acid and ethylene. AT1G15890, homolog of Brapa_ESTC034248 encodes putative CC-NBS-LRR class protein that causes bacterial cell death (Yang et al. 2016). AT3G50950, homolog of Brapa_ESTC036172, encodes Arabidopsis R protein HOPZ-activated resistance 1 (ZAR1) required for recognition of HopZ1a, Pseudomonas syringae type III secreted effector (Lewis et al. 2010). Examination of expression pattern and sequence variations in 17 disease resistance genes, especially homologs of above mentioned three Arabidopsis genes, could be useful to develop molecular markers for disease resistance in Brassica species. 
Table 4 NBS class defense-related genes showing high expression in $F_{1}$ hybrids at least two growing stages

\begin{tabular}{|c|c|c|c|c|c|c|c|c|c|c|c|c|}
\hline \multirow{3}{*}{ At_Locus } & \multirow{3}{*}{$\begin{array}{c}\text { Description, blastN } \\
\text { (Disease Resistant Protein) }\end{array}$} & \multirow{3}{*}{ Br_SEQ_ID } & \multirow{3}{*}{ BRAD_ID } & \multicolumn{9}{|c|}{ Probe intensity } \\
\hline & & & & \multicolumn{3}{|c|}{$15 \mathrm{DAG}$} & \multicolumn{3}{|c|}{$30 \mathrm{DAG}$} & \multicolumn{3}{|c|}{$60 \mathrm{DAG}$} \\
\hline & & & & 25080 & 25081 & 25083 & 25080 & 25081 & 25083 & 25080 & 25081 & 25083 \\
\hline AT1G53350 & CC-NBS-LRR class, putative & Brapa_ESTC015346 & $\mathrm{Bra} 037448$ & 277 & 4520 & 2420 & 406 & 1119 & 2610 & 625 & 6549 & 1265 \\
\hline AT1G72890 & TIR-NBS class, putative & Brapa_ESTC036158 & $\mathrm{Bra} 016029$ & 246 & 280 & 10 & 175 & 373 & 54 & 328 & 236 & 52 \\
\hline \multirow[t]{3}{*}{ AT5G18350 } & TIR-NBS-LRR class, putative & Brapa_ESTC037594 & $\mathrm{Bra} 001161$ & 886 & 1749 & 990 & 766 & 838 & 526 & 628 & 764 & 302 \\
\hline & & Brapa_ESTC042689 & $\mathrm{Bra} 001160$ & 5848 & 5689 & 4363 & 4273 & 4893 & 3867 & 3076 & 4010 & 2814 \\
\hline & & Brapa_ESTC029890 & $\mathrm{Bra} 006487$ & 279 & 417 & 243 & 261 & 304 & 324 & 176 & 468 & 378 \\
\hline \multirow[t]{2}{*}{ AT5G11250* } & TIR-NBS-LRR class, putative & Brapa_ESTC032144 & $\mathrm{Bra} 006146$ & 2851 & 3805 & 2565 & 1739 & 2010 & 1919 & 1608 & 2204 & 1326 \\
\hline & & Brapa_ESTC009435 & $\mathrm{Bra} 006556$ & 3459 & 3886 & 3750 & 2819 & 3352 & 2620 & 3486 & 3439 & 3281 \\
\hline \multirow[t]{2}{*}{ AT5G66900 } & CC-NBS-LRR class, putative & Brapa_ESTC008403 & $\mathrm{Bra} 025290$ & 867 & 2003 & 1682 & 1430 & 1837 & 1437 & 1491 & 1783 & 1162 \\
\hline & & Brapa_ESTC025668 & $\mathrm{Bra} 018057$ & 9875 & 12594 & 10126 & 8353 & 9561 & 11576 & 4918 & 6728 & 4967 \\
\hline AT5G46450 & TIR-NBS-LRR class, putative & Brapa_ESTC046013 & Bra022036? & 194 & 108 & 28 & 107 & 118 & 83 & 87 & 164 & 113 \\
\hline AT5G41540 & TIR-NBS-LRR class, putative & Brapa_ESTC018846 & $\mathrm{Bra} 001162$ & 3595 & 3731 & 1929 & 2624 & 2481 & 1933 & 2191 & 2890 & 2021 \\
\hline AT1G15890* & CC-NBS-LRR class, putative & Brapa_ESTC034248 & $\mathrm{Bra} 018835$ & 5532 & 4963 & 4243 & 5609 & 6472 & 5486 & 3854 & 4051 & 3145 \\
\hline AT1G69550 & TIR-NBS class, putative & Brapa_ESTC034328 & $\mathrm{Bra} 020936$ & 65 & 119 & 132 & 95 & 169 & 102 & 54 & 164 & 134 \\
\hline AT4G27190 & NBS-LRR class, putative & Brapa_ESTC008807 & $\mathrm{Bra} 026368$ & 3914 & 4404 & 4023 & 6671 & 8044 & 5932 & 6100 & 5244 & 4694 \\
\hline AT5G22690 & TIR-NBS-LRR class, putative & Brapa_ESTC021828 & $\mathrm{Bra} 034079$ & 1499 & 1075 & 1727 & 1629 & 3193 & 2402 & 923 & 1369 & 1278 \\
\hline AT3G50950* & CC-NBS-LRR class, putative & Brapa_ESTC036172 & Bra036845 & 17874 & 13266 & 11186 & 11327 & 11777 & 10183 & 7026 & 8846 & 8697 \\
\hline AT5G41750 & TIR-NBS-LRR class, putative & Brapa_ESTC042275 & $\mathrm{Bra} 013144$ & 9878 & 10607 & 8205 & 1520 & 1995 & 1716 & 331 & 384 & 1336 \\
\hline
\end{tabular}

Table 5 Select genes for the RT-PCR experiment with additive or non-additive expression; f, high PI value in female parent; m, high PI value in male parent; +, higher PI value in F1 hybrid outside the parental values; -, lower PI value in F1 compared to parental values

\begin{tabular}{|c|c|c|c|c|c|c|c|c|c|}
\hline \multirow{3}{*}{$\begin{array}{c}\text { Gene } \\
\text { expression } \\
\text { pattern }\end{array}$} & \multirow{3}{*}{ Stage } & \multirow{3}{*}{$B r \_$SEQ ID } & \multirow{3}{*}{ At_Locus } & \multirow{3}{*}{ Description, blastN } & \multicolumn{4}{|c|}{ PI value } & \multirow{3}{*}{ Remarks } \\
\hline & & & & & 25080 & 25081 & 25083 & $\begin{array}{l}\text { Mid-parent } \\
\text { value }\end{array}$ & \\
\hline & & & & & Female & F1 hybrid & Male & $(80+83) / 2$ & \\
\hline \multirow{7}{*}{$\begin{array}{l}\text { Additive } \\
\text { expression }\end{array}$} & $15 \mathrm{DAG}$ & Brapa_ESTC034378 & AT3G10690 & DNA gyrase subunit A family protein & 7362.4 & 5731.5 & 4099.3 & 5730.8 & $f$ \\
\hline & & Brapa_ESTC011594 & AT5G11110 & ATSPS2F (sucrose phosphate synthase 2F) & 473.5 & 1011.8 & 1547.8 & 1010.7 & $\mathrm{~m}$ \\
\hline & $30 \mathrm{DAG}$ & Brapa_ESTC012536 & AT4G01310 & Ribosomal protein L5 family protein & 18408.4 & 15713.8 & 13017.2 & 15712.8 & f \\
\hline & & Brapa_ESTC022326 & AT1G27190 & LRR transmembrane protein kinase, putative & 9591.3 & 8719.6 & 7847.5 & 8719.4 & f \\
\hline & & Brapa_ESTC005539 & AT4G29010 & AIM1 (ABNORMAL INFLORESCENCE MERISTEM) & 1554.5 & 2467.4 & 3379.4 & 2466.9 & $\mathrm{~m}$ \\
\hline & $60 \mathrm{DAG}$ & Brapa_ESTC046155 & AT5G46630 & $\begin{array}{l}\text { Clathrin adaptor complexes medium subunit family } \\
\text { protein }\end{array}$ & 17451.9 & 15204.9 & 12958.5 & 15205.2 & f \\
\hline & & Brapa ESTC050491 & AT2G39730 & RCA (RUBISCO ACTIVASE) & 11021.2 & 9163.8 & 7307.6 & 9164.4 & $f$ \\
\hline \multirow{9}{*}{$\begin{array}{c}\text { Non-additive } \\
\text { expression }\end{array}$} & $15 \mathrm{DAG}$ & Brapa_ESTC011189 & AT2G05100 & $\begin{array}{l}\text { LHCB2.1 (Photosystem II light harvesting complex } \\
\text { gene 2.1) }\end{array}$ & 39951.7 & 45713.9 & 39491.5 & 39721.6 & + \\
\hline & & Brapa_ESTC016074 & AT1G29930 & CAB1 (CHLOROPHYLL A/B BINDING PROTEIN 1) & 51284.7 & 44655.3 & 55324.5 & 53304.6 & - \\
\hline & & Brapa_ESTC025522 & AT5G66190 & $\begin{array}{l}\text { ATLFNR1 (LEAF FNR 1); NADPH dehydrogenase/ } \\
\text { oxidoreductase }\end{array}$ & 36986.1 & 38352.5 & 27943.2 & 32464.6 & + \\
\hline & $30 \mathrm{DAG}$ & Brapa_ESTC047891 & AT1G20620 & CAT3 (CATALASE 3) & 38890.8 & 51640.6 & 45377.4 & 217134.1 & + \\
\hline & & Brapa_ESTC032082 & AT5G09440 & Phosphate-responsive protein, putative & 12234.3 & 28554.8 & 16204.8 & 14219.6 & + \\
\hline & & Brapa_ESTC016880 & AT2G26230 & Uricase / urate oxidase / nodulin 35 , putative & 6084.3 & 17723.4 & 14794.1 & 10439.2 & + \\
\hline & & Brapa_ESTC021056 & AT5G25540 & CID6 (CTC-Interacting Domain 6); protein binding & 16785.4 & 17366.5 & 8675.0 & 12730.2 & + \\
\hline & $60 \mathrm{DAG}$ & Brapa_ESTC022798 & AT1G06680 & $\begin{array}{l}\text { PSBP-1 (OXYGEN-EVOLVING ENHANCER } \\
\text { PROTEIN 2) }\end{array}$ & 39278.9 & 50232.5 & 39308.9 & 39293.9 & + \\
\hline & & Brapa_ESTC013408 & AT5G04590 & SIR (sulfite reductase) & 6239.6 & 14146.4 & 4784.8 & 5512.2 & + \\
\hline
\end{tabular}

Expression profiling of selected genes

To confirm microarray results, several classes of genes were selected and subjected to RT-PCR (Fig. 3). Gene description and PI values were presented in Table 2 for up-regulated genes at all three stages and Table 5 for additively and non-additively expressed genes in F1 plants. Although there were some variatinos were detected, RT-PCR signals were similar to that observed with PI values, indicating that microarray experiments reliably reflected transcription levels in general. Particularly, all up-regulated genes were confirmed to be predominantly expressed in $F_{1}$ hybrids, while genes 

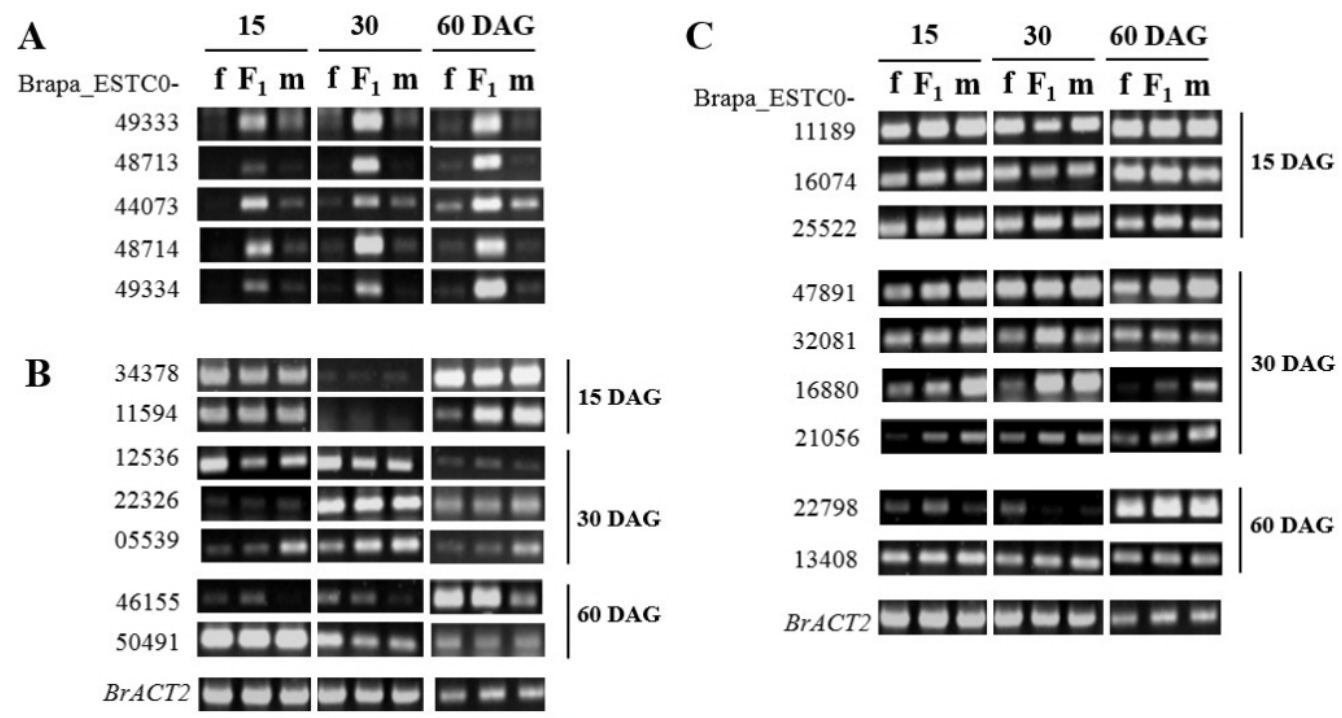

Fig. 3 RT-PCR confirmation of expression patters for selected genes. A, Up-regulated genes in F1 hybrid at all stages; B, Genes with additive expression at the indicated stage (right side); $\mathrm{C}$, Genes with non-additive expression at the indicated stage (right side)

showing either additive or non-additive expression displayed stage-specific expression patterns. Since all up-regulated genes in Fig. 3A have not been functionally characterized, the identification of these genes by heterosis-related expression pattern provides a good starting point to characterize their functions, such as growth and photosynthesis.

In conclusion, $F_{1}$ hybrids generated by crosses between genetically distinct individuals show heterosis phenotypes through complex mechanisms, in terms of numbers of genes and timing of their actions. Multiple loci seem to be involved in heterosis for different traits and in different hybrids (Schnable and Springer 2013). Identification of genes governing heterosis mechanism is therefore very challenging. We have obtained valuable information from $\mathrm{Br} 300 \mathrm{~K}$ microarray by comparing gene expression patterns in $\mathrm{F}_{1}$ hybrid and its parents. We identified (1) several putative HRGs that are highly up-regulated in $\mathrm{F}_{1}$ hybrids and possibly responsible for heterosis phenotype and (2) some B. rapa homologs of Arabidopsis HRGs promising to play similar roles in non-heading Chinese cabbage, and (3) several NBSLRR class genes showing heterosis-related expression and possibly involved in defense signaling. Our finding will facilitate to improve hybrid breeding and to develop molecular markers for the disease resistance.

\section{Acknowledgement}

This work was supported by Research Fund of Chungnam National University (CNU), Daejeon, Korea, to Yoonkang Hur (2015-1115-01).

\section{Supplementary materials}

Supplementary Table 1 List of primer sequences used in RT-PCR Supplementary Table 2 Br300K microarray results annotated with Arabidopsis TAIR7.

\section{References}

Auger DL, Gray AD, Ream TS, Kato A, Coe EH Jr, Birchler JA (2005) Nonadditive gene expression in diploid and triploid hybrids of maize. Genetics 169:389-397

Baranwal VK, Mikkilineni V, Zehr UB, Tyagi AK, Kapoor S (2012) Heterosis: emerging ideas about hybrid vigour. J Exp Bot 63:6309-6314

Basunanda P, Radoev M, Ecke W, Friedt W, Becker HC, Snowdon RJ (2010) Comparative mapping of quantitative trait loci involved in heterosis for seedling and yield traits in oilseed rape (Brassica napus L.). Theor Appl Genet 120: 271-281

Charlesworth D, Willis JH (2009) The genetics of inbreeding depression. Nat Rev Genet 10:783-96

Chen ZF (2013) Genomic and epigenetic insights into the molecular bases of heterosis. Nat Rev Genet 14:471-482

Dong X, Feng H, Xu M, Lee J, Kim YK, Lim YP, Piao Z, Park YD, Ma H, Hur Y (2013) Comprehensive analysis of genic male sterility-related genes in Brassica rapa using a newly developed Br300K oligomeric chip. PLoS One 8: e 72178

Eitas TK, Nimchuk ZL, Dangl JL (2008) Arabidopsis TAO1 is a TIR-NB-LRR protein that contributes to disease resistance induced by the Pseudomonas syringae effector AvrB. Proc Natl Acad Sci USA. 105:6475-6480

Fu D, Xiao M, Hayward A, Jiang G, Zhu L, Zhou Q, Li J, Zhang M (2015) What is crop heterosis: new insights into an old topic. J Appl Genetics 56:1-13 
Greaves IK, Gonzalez-Bayon R, Wang L, Zhu A, Liu PC, Groszmann M, Peacock WJ, Dennis ES (2015) Epigenetic changes in hybrids. Plant Physiol 168:1197-1205

Groszmann M, Greaves IK, Albertyn ZI, Scofield GN, Peacock WJ, Dennis ES (2011) Hormone-regulated defense and stress response networks contribute to heterosis in Arabidopsis $\mathrm{F}_{1}$ hybrids. Proc Natl Acad Sci USA 112:E6397-E6406

Groszmann M, Greaves IK, Fujimoto R, Peacock WJ, Dennis ES (2013) The role of epigenetics in hybrid vigour. Trends Genet 29:684-690

Guo M, Rupe MA, Yang X, Crasta O, Zinselmeier C, Smith OS, Bowen B (2006) Genome-wide transcript analysis of maize hybrids: allelic additive gene expression and yield heterosis. Theor Appl Genet 113:831-845

Hatsugai N, Hillmer R, Yamaoka S, Hara-Nishimura I, Katagiri F (2016) The $\mu$ subunit of Arabidopsis adaptor protein-2 is involved in effector-triggered immunity mediated by membranelocalized resistance proteins. Mol Plant Microbe Interact 29:345-351

Hochholdinger F, Hoecker N (2007) Towards the molecular basis of heterosis. Trends Plant Sci 12:427-432

Hou XL, Cao SC, Zhang SN, Zhang ZC, Wang JJ, Sun HX (2005) Selection of non-heading Chinese cabbage cultivar Shulü with high-quality. J Nanging Agr Univ 28:30-33

Irizarry RA, Hobbs B, Collin F, Beazer-barclay YD, Antonellis KJ, Scherf U, Speed TP (2003) Exploration, normalization, and summaries of high density oligonucleotide array probe level data. Biostatistics 4:249264

Jeong SY, Ahmed NU, Jung HJ, Kim HT, Park JI, Nou IS (2017) Discovery of candidate genes for heterosis breeding in Brassica oleracea L. Acta Physiol Plant 39:180

Kawanabe T, Ishikura S, Miyaji N, Sasaki T, Wu LM, Itabashi E, Takada S, Shimizu M, Takasaki-Yasuda T, Osabe K, Peacock WJ, Dennis ES, Fujimoto R. (2016) Role of DNA methylation in hybrid vigor in Arabidopsis thaliana. Proc Natl Acad Sci USA. 113: E6704-E6711

Kim GT, Shoda K, Tsuge T, Cho KH, Uchimiya H, Yokoyama R, Nishitani K, Tsukaya H (2002) The ANGUSTIFOLIA gene of Arabidopsis, a plant $C t B P$ gene, regulates leaf-cell expansion, the arrangement of cortical microtubules in leaf cells and expression of a gene involved in cell-wall formation. EMBO J 21:1267-1279

Ko DK, Rohozinski D, Song Q, Taylor SH, Juenger TE, Harmon FG, Chen ZJ (2016) Temporal shift of circadian-mediated gene expression and carbon fixation contributes to biomass heterosis in maize hybirds. PLoS Genet 12:e1006197

Kumar S, Kanakachari M, Gurusamy D, Kumar K, Narayanasamy P, Kethireddy Venkata P, Solanke A, Gamanagatti S, Hiremath V, Katageri IS, Leelavathi S, Kumar PA, Reddy VS (2016) Genome-wide transcriptomic and proteomic analyses of bollworm-infested developing cotton bolls revealed the genes and pathways involved in the insect pest defense mechanism. Plant Biotechnol J 14:1438-1455

Lewis JD, Wu R, Guttman DS, Desveaux D (2010) Allele-specific virulence attenuation of the Pseudomonas syringae HopZ1a type III effector via the Arabidopsis ZAR1 resistance protein. PLoS Genet 6:e1000894

Li A, Fang MD, SongWQ, Chen CB, Qi LW, Wang CG (2012) Gene expression profiles of two intraspecific Larix lines and their reciprocal hybrids. Mol Biol Rep 39:3773-3784

Li D, Huang Z, Song S, Xin Y, Mao D, Lv Q, Zhou M, Tian D, Tang M, Wu Q, Liu X, Chen T, Song X, Fu X, Zhao B, Liang C, Li A, Liu G, Li S, Hu S, Cao X, Yu J, Yuan L, Chen C, Zhu L (2016) Integrated analysis of phenome, genome, and transcriptome of hybrid rice uncovered multiple heterosis-related loci for yield increase. Proc Natl Acad Sci USA 113: E6026-E6035

Lippman ZB, Zamir D (2007) Heterosis: revisiting the magic. Trends Genet 23:60-66

Meyer S, Pospisil H, Scholten S (2007) Heterosis associated gene expression in maize embryos 6 days after fertilization exhibits additive, dominant and overdominant pattern. Plant Mol Biol 63:381-391

Miller M, Song Q, Shi X, Juenger TE, Chen ZJ (2015) Natural variation in timing of stress-responsive gene expression predicts heterosis in intraspecific hybrids of Arabidopsis. Nat Commun 6:7453

Ni Z, Kim ED, Ha M, Lackey E, Liu J, Zhang Y, Sun Q, Chen ZJ (2009) Altered circadian rhythms regulate growth vigour in hybrids and allopolyploids. Nature 457:327-331

Plötner B, Nurmi M, Fischer A, Watanabe M, Schneeberger K, Holm S, Vaid N, Schöttler MA, Walther D, Hoefgen R, Weigel D, Laitinen RAE (2017) Chlorosis caused by two recessively interacting genes reveals a role of RNA helicase in hybrid breakdown in Arabidopsis thaliana. Plant J 91:251-262

Romagnoli S, Maddaloni M, Livini C, Motto M (1990) Relationship between gene expression and hybrid vigor in primary root tips of young maize (Zea mays L) plantlets. Theor Appl Genet 80:767-775

Saeki N, Kawanabe T, Ying H, Shimizu M, Kojima M, Abe H, Okazaki K, Kaji M, Taylor JM, Sakakibara H, Peacock WJ, Dennis ES, Fujimoto R (2016) Molecular and cellular characteristics of hybrid vigour in a commercial hybrid of Chinese cabbage. BMC Plant Biol 16:45

Sarazin V, Duclercq J, Mendou B, Aubanelle L, Nicolas V, Aono M, Pilard S, Guerineau F, Sangwan-Norreel B, Sangwan RS (2015) Arabidopsis BNT1, an atypical TIR-NBS-LRR gene, acting as a regulator of the hormonal response to stress. Plant Sci 239:216-229

Schnable PS, Springer NM (2013) Progress toward understanding heterosis in crop plants. Annu Rev Plant Biol 64:71-88

Shen H, He H, Li J, Chen W, Wang X, Guo L, Peng Z, He G, Zhong S, Qi Y, Terzaghi W, Deng XW (2012) Genome-wide analysis of DNA methylation and gene expression changes in two Arabidopsis ecotypes and their reciprocal hybrids. Plant Cell 24:875-892

Song H, Yi H, Do c, Han CT, Nou IS, Hur Y (2017) Genome-wide analysis of gene expression to distinguish photoperiod-dependent and -independent flowering in Brassicaceae. Genes Genom 39:207-223 
Stupar RM, Springer NM (2006) Cis-transcriptional variation in maize inbred lines B73 and Mo17 leads to additive expression patterns in the $F_{1}$ hybrid. Genetics 173:2199-2210

Stupar RM, Gardiner JM, Oldre AG, Haun WJ, Chandler VL, Springer NM (2008) Gene expression analyses in maize inbreds and hybrids with varying levels of heterosis. BMC Plant Biol 8:33

Tsaftaris AS (1995) Molecular aspects of heterosis in plants. Physiol Plant 94:362-370

Wang L, Wu LM, Greaves IK, Zhu A, Dennis ES, Peacock WJ (2017) PIF4-controlled auxin pathway contributes to hybrid vigor in Arabidopsis thaliana. Proc Natl Acad Sci USA 114:E3555-E3562

Wang T, Sui Z, Liu X, Li Y, Li H, Xing J, Song F, Zhang Y, Sun Q, $\mathrm{NiZ}$ (2016) Ectopic expression of a maize hybrid up-regulated gene, ErbB-3 binding Protein 1 (ZmEBP1), increases organ size by promoting cell proliferation in Arabidopsis. Plant Sci 243:23-34

Workman C, Jensen LJ, Jarmer H, Berka R, Gautier L, Nielser HB, Saxild HH, Nielsen C, Brunak S, Knudsen S (2002) A new non-linear normalization method for reducing variability in DNA microarray experiments. Genome Biol 3:research0048

Yang Y, Wu X, Xuan H, Gao Z (2016) Functional analysis of plant NB-LRR gene $L 3$ by using E. coli. Biochem Biophys Res Commun 478:1569-1574

Zemach A, Kim MY, Hsieh PH, Coleman-Derr D, Eshed-Williams L, Thao K, Harmer SL, Zilberman D (2013) The Arabidopsis nucleosome remodeler DDM1 allows DNA methyltransferases to access H1-containing heterochromatin. Cell 153:193-205 